\title{
NanoMIP-Based Solid Phase Extraction of Fluoroquinolones from Human Urine: A Proof-of-Concept Study
}

\author{
Matteo Chiarello (D), Laura Anfossi (D), Simone Cavalera (D, Fabio Di Nardo (D), Thea Serra \\ and Claudio Baggiani *(D)
}

check for updates

Citation: Chiarello, M.; Anfossi, L.; Cavalera, S.; Di Nardo, F.; Serra, T.; Baggiani, C. NanoMIP-Based Solid Phase Extraction of Fluoroquinolones from Human Urine: A Proof-of-Concept Study. Separations 2021, 8, 226. https://doi.org/ 10.3390 /separations 8110226

Academic Editor:

Antonio Martin-Esteban

Received: 27 October 2021

Accepted: 16 November 2021

Published: 19 November 2021

Publisher's Note: MDPI stays neutral with regard to jurisdictional claims in published maps and institutional affiliations.

Copyright: (c) 2021 by the authors. Licensee MDPI, Basel, Switzerland. This article is an open access article distributed under the terms and conditions of the Creative Commons Attribution (CC BY) license (https:/ / creativecommons.org/licenses/by/ $4.0 /)$.
Department of Chemistry, University of Torino, 10125 Torino, Italy; matteo.chiarello@unito.it (M.C.); laura.anfossi@unito.it (L.A.); simone.cavalera@unito.it (S.C.); fabio.dinardo@unito.it (F.D.N.); thea.serra@unito.it (T.S.)

* Correspondence: claudio.baggiani@unito.it

\begin{abstract}
NanoMIPs that are prepared by solid phase synthesis have proven to be very versatile, but to date only limited attention has been paid to their use in solid phase extraction. Thus, since nanoMIPs show close similarities, in terms of binding behavior, to antibodies, it seems relevant to verify if it is possible to use them as mimics of the natural antibodies that are used in immunoextraction methods. As a proof-of-concept, we considered prepared nanoMIPs against fluoroquinolone ciprofloxacin. Several nanoMIPs were prepared in water with polymerization mixtures of different compositions. The polymer with the highest affinity towards ciprofloxacin was then grafted onto a solid support and used to set up a solid phase extraction-HPLC method with fluorescence detection, for the determination of fluoroquinolones in human urine. The method resulted in successful selection for the fluoroquinolone antibiotics, such that the nanoMIPs were suitable for direct extraction of the antibiotics from the urine samples at the $\mu \mathrm{g} \mathrm{mL}^{-1}$ level. They required no preliminary treatment, except for a $1+9(v / v)$ dilution with a buffer of $\mathrm{pH} 4.5$ and they had good analyte recovery rates; up to $85 \%$ with precision in the range of 3 to $4.5 \%$, without interference from the matrix. These experimental results demonstrate, for the first time, the feasibility of the use of nanoMIPs to develop solid phase extraction methods.
\end{abstract}

Keywords: molecularly imprinted polymer; nanoMIP; solid phase synthesis; MISPE; solid phase extraction; fluoroquinolone; ciprofloxacin; urine

\section{Introduction}

Molecularly imprinted polymers (MIPs) find in the so-called "molecularly imprinted solid phase extraction" (MISPE) technique one of their most popular applications, consisting of selectively extracting target analytes in the presence of interfering substances and complex matrices. Whether in the form of cartridges [1], monoliths [2], magnetic particles [3], or nanofibers [4], the application of MIPs to extraction problems is certainly competitive due to their resistance to chemical and biological degradation, the versatility of their applications and the operative costs (if compared to similar methods based on natural receptors such as immunoaffinity extraction) $[5,6]$.

Despite these advantageous characteristics, the MISPE technique shows several drawbacks which limit its wider applicability. First of all, the preparation of the imprinted material requires the introduction into the polymerization mixture of a fair amount of the target molecule that must act as a template [7]. Thus, the preparation of an adequate quantity of the polymer requires a large quantity of the template, which cannot always be recovered and recycled. In the case of analytes that are difficult to find, expensive or unstable, or those that represent a hazard to health or safety, this is a practical obstacle that cannot be easily overcome. Furthermore, the complete removal of the template from the imprinted polymer is frequently rather difficult $[8,9]$ and the unextracted residues can 
slowly leach out in the so-called "bleeding effect", contaminating the samples in the solid phase extraction process and irreparably compromising the results of the analysis.

To overcome these drawbacks, the "mimic template" technique has been introduced, where a putative molecule that is structurally similar to the target molecule is able to raise imprinted binding sites but does not interfere with the subsequent analytical technique. This approach avoids the bleeding effect, but still requires the availability of large quantities of the mimic template, which frequently must be prepared by ad hoc synthesis [10].

An innovative approach to solving all of these issues is presented by the solid phase synthesis of nanoMIPs [11,12]. In this process, illustrated in Scheme 1, the polymerization process takes place in the interstitial space between packed glass beads that are covalently grafted with template molecules. The growth of cross-linked polymeric chains takes place in proximity to the glass beads' surfaces, resulting in the imprinting of the nascent nanoparticles by the grafted template molecules. At the end of the polymerization process, any residual monomers, polymerization by-products and low-affinity polymers are washed away, whilst the high-affinity nanoMIPs are recovered by washing with a solution capable of breaking the non-covalent molecular interactions between the imprinted nanoparticles and the grafted template molecules. This solid phase synthesis technique addresses many of the drawbacks of traditional solution-synthesis techniques. In fact, because the template molecules are covalently grafted onto the glass beads, no residual template molecules are present in the nanoMIPs, avoiding the bleeding effect entirely. Moreover, as the functionalized glass beads require a small amount of the template and can be cleaned and reused many times, this approach allows the use of expensive templates. Additionally, in the case of toxic or harmful substances, the confinement of the reaction to the glass beads' surfaces eliminates any health risks during the manipulation of the solid phase [13].

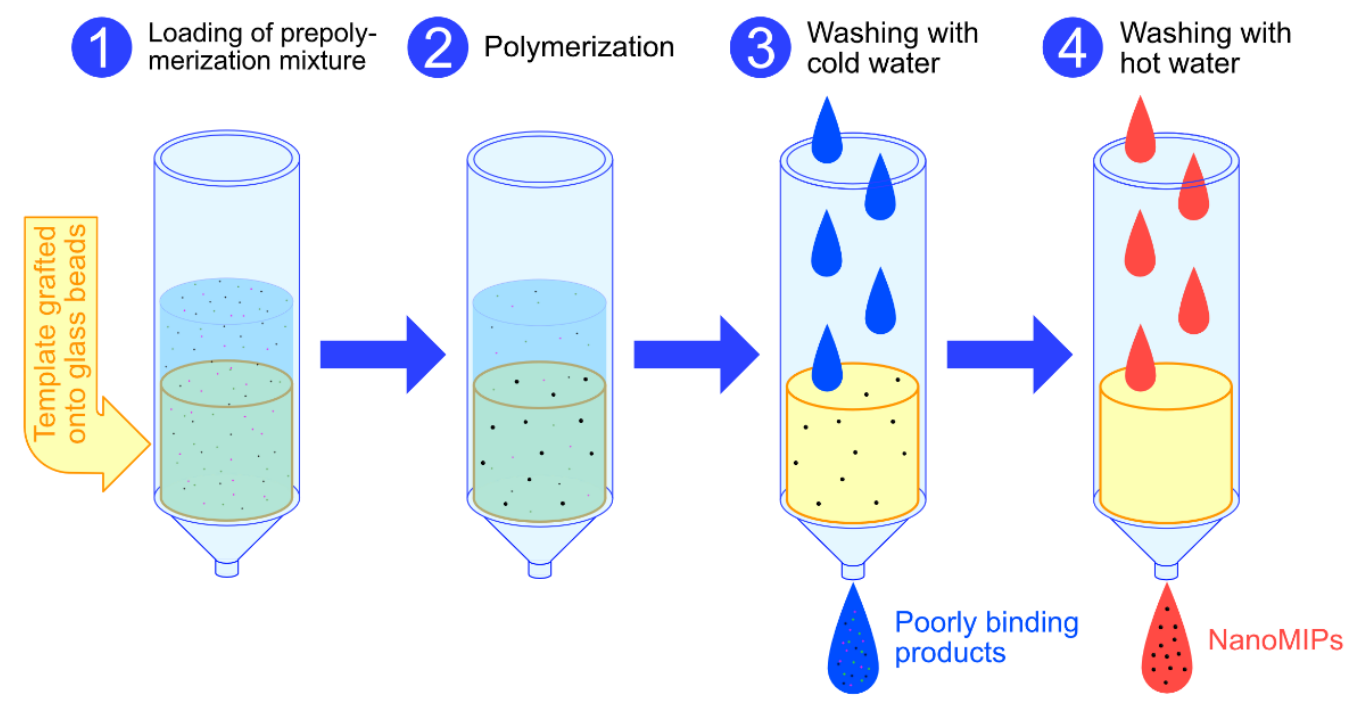

Scheme 1. Preparation of nanoMIPs by solid phase synthesis method.

Solid phase synthesis has proved to be very versatile and nanoMIPs that are able to target small molecules [14,15], macrocyclic antibiotics [16,17], toxins [18,19], and peptides $[20,21]$ have been described, and used, for the development of sensors and biomimetic assays. However, to date only limited attention has been paid to the use of nanoMIPs in solid phase extraction [14]. Therefore, since nanoMIPs show close similarities, in terms of binding behavior, to natural antibodies, it seems relevant to verify if it is possible to use them as substitutes for natural antibodies in immunoextraction methods.

As a proof-of-concept, we considered prepared nanoMIPs against ciprofloxacin, a fluoroquinolone of relevant interest as an analytical target in MISPE [22-24] on which we have recently published the characterization of the binding properties $[25,26]$. Several nanoMIPs were prepared with polymerization mixtures of different compositions and the 
polymer with the highest affinity towards ciprofloxacin was used to set up a method for the extraction of the target analyte from human urine.

\section{Materials and Methods}

\subsection{Materials}

The template molecule, ciprofloxacin hemisuccinamide (CIP-HS), was synthesized according to a modified version of the procedure given by Noël et al. [27]. Glass beads (Spheriglass-2429, 70-100 $\mu \mathrm{m}$ average particle size, Potters, UK) were aminated and grafted with the template as previously described [26]. The acrylic acid (AA), chlortetracycline (CTX), ciprofloxacin (CIP), danofloxacin (DAN), enrofloxacin (ENR), $\mathrm{N}$-isopropylacrylamide (NIPAm), levofloxacin (LEV), lomefloxacin (LOM), N,N'-methylene-bis-acrylamide (BIS), morpholinoethansulfonic acid (sodium salt, MES), moxifloxacin (MOX), norfloxacin (NOR), sarafloxacin (SAR), N-tert-butylacrylamide (TBAm), ammonium persulphate (APS) and $\mathrm{N}, \mathrm{N}, \mathrm{N}^{\prime}, \mathrm{N}^{\prime}$-tetramethylethylenediamine (TEMED) that were used in this study were purchased from Merck (Milan, Italy). The solvents and all other chemicals were also purchased from Merck (Milan, Italy). All of the solvents were of HPLC grade, whereas all of the chemicals were of analytical grade. The water used was ultra-purified in a PURELAB Prima System from Elga (Marlow, UK). The fluoroquinolone stock solutions were prepared by dissolving $25 \mathrm{mg}$ of the substance in $25 \mathrm{~mL}$ of water/methanol $1+1(v / v)$, these solutions were then stored in the dark at $-20{ }^{\circ} \mathrm{C}$.

Synthetic urine was prepared as previously reported [28], stored at $4{ }^{\circ} \mathrm{C}$ and discarded no later than a week after its preparation.

\subsection{Synthesis of NanoMIPs}

The polymerization mixtures were prepared by modifying the general protocol reported in the literature [13] and adjusting the dilution of the monomers in order to avoid the formation of unwanted lumps of polymer. All of the mixtures (the molar compositions of which are reported in Table S1, Supplementary Materials) were made in $25 \mathrm{~mL}$ of ultrapure water by mixing BIS, AA, NIPAm and TBAm (each of which was dissolved in $0.5 \mathrm{~mL}$ of ethanol). Then, $5 \mathrm{~mL}$ of each of the mixtures was added to $50 \mathrm{~mL}$ capacity polypropylene SPE cartridges containing $2.5 \mathrm{~g}$ of functionalized glass beads. The cartridges were purged with nitrogen for $5 \mathrm{~min}, 3 \mu \mathrm{L}$ of TEMED and $100 \mu \mathrm{L}$ of a $30 \mathrm{mg} \mathrm{mL}^{-1}$ aqueous solution of APS were added, and then polymerization was carried out at room temperature for $1 \mathrm{~h}$ in a roller-equipped incubator. The supernatant was drained by vacuum aspiration, the dry cartridges were cooled to $4{ }^{\circ} \mathrm{C}$ and the polymerization by-products and low-affinity nanoMIPs were washed with $10 \times 2 \mathrm{~mL}$ of ice-cold water. The high-affinity nanoMIPs were collected by eluting the cartridges with $5 \times 2 \mathrm{~mL}$ of hot water. The eluates were lyophilized, weighed and stored at $4{ }^{\circ} \mathrm{C}$.

The nanoMIPs were grafted onto aminated glass beads in accordance with the protocol that has been previously reported [26].

\subsection{HPLC Method}

A reverse-phase HPLC analysis was used for fluoroquinolone determination. The HPLC apparatus (Merck-Hitachi, Milan, Italy) that was utilized was a LaChrom Elite system, composed of a programmable binary pump (L-2130), an auto-sampler (L-2200) and a fluorescence detector (L-7485). The LaChrom Elite system was provided with EZChrom Elite software for instrumental programming, data acquisition and data processing. The column used was a $100 \mathrm{~mm} \times 4.6 \mathrm{~mm}$ C-18 Onyx (Phenomenex, Milan, Italy). The mobile phases used were water/acetonitrile $88+12$, acetic acid $1 \%(v / v)$ for fluoroquinolones, a $50 \mathrm{mmol} \mathrm{L}^{-1}$ acetate buffer and $\mathrm{pH} 8.1 /$ methanol $40+60(v / v)$ for chlortetracycline. Elution was performed in isocratic conditions at a flow rate of $0.7 \mathrm{~mL} \mathrm{~min}^{-1}$. The sample volume that was injected was $5 \mu \mathrm{L}$ and the fluorescence wavelengths were: CIP, DAN, ENR, LOM, NAR, SAR: $\lambda_{\mathrm{ex}}=280 / \lambda_{\mathrm{em}}=440 \mathrm{~nm} ; \mathrm{LEV}: \lambda_{\mathrm{ex}}=278 / \lambda_{\mathrm{em}}=540 \mathrm{~nm}$; MOX: $\lambda_{\mathrm{ex}}=294 / \lambda_{\mathrm{em}}=503 \mathrm{~nm}$; and CTX: $\lambda_{\mathrm{ex}}=380 / \lambda_{\mathrm{em}}=532 \mathrm{~nm}$. The analyte solutions 
between 5 and $100 \mathrm{ng} \mathrm{mL}^{-1}$ in concentration were analyzed in triplicate and the mean peak areas were plotted against each concentration. The calibration plot was drawn by using a weighted linear regression (weight $=1$ / conc).

\subsection{Determination of Binding Properties}

To measure the equilibrium binding isotherms, unbound fractions of fluoroquinolones were measured by reverse-phase HPLC analysis with fluorescence detection, in accordance with the previous literature [25]. Each experimental data point was assessed as the average of three repeated measures and binding parameters were calculated in accordance with the Langmuir binding isotherm by nonlinear least squares fitting.

\subsection{Preparation of MISPE Cartridges}

Adequate amounts of glass beads grafted with nanoMIPs $(1 \mathrm{~g})$ were suspended in water and packed into $5 \mathrm{~mL}$ capacity, empty polypropylene SPE cartridges that were provided with frits to secure the packing and outlet stopcocks. The columns were connected to a vacuum manifold and washed extensively with water, then dried under a vacuum. Immediately before any use, the cartridges were activated with $500 \mu \mathrm{L}$ of MES buffer $50 \mathrm{mmol} \mathrm{L}^{-1}, \mathrm{pH} 4.5$. When necessary, the columns were cleaned and regenerated by washing with $500 \mu \mathrm{L} \mathrm{mL}$ of water/acetic acid $9+1 v / v$ and $3 \times 500 \mu \mathrm{L}$ of water.

\subsection{Optimization of the MISPE Method}

In order to optimize the procedure of fluoroquinolone extraction, different protocols were applied during the loading and washing steps. In the subsequent experiments, each extraction was repeated three times and the amount of analyte recovery was evaluated as the average of the single values that were measured.

To investigate the effects of the different loading solutions, ciprofloxacin $\left(200 \mathrm{ng} \mathrm{mL}^{-1}\right)$ in synthetic urine was diluted $1+9 v / v$ with $50 \mathrm{mmol} \mathrm{L}^{-1}$ MES (pH 4.5-5.5) or phosphate buffers ( $\mathrm{pH}$ 6.5-8.5), and then $250 \mu \mathrm{L}$ of diluted solution was loaded into the cartridge. After the sample loading, the cartridge was washed with $250 \mu \mathrm{L}$ of methanol/acetic acid $9+1 v / v$.

To investigate the effects of the different washing solutions, ciprofloxacin $\left(200 \mathrm{ng} \mathrm{mL}^{-1}\right)$ in synthetic urine was diluted $1+9 v / v$ with $50 \mathrm{mmol} \mathrm{L}^{-1}$ MES buffer, $\mathrm{pH} 4.5$, then $250 \mu \mathrm{L}$ of diluted solution was loaded into the cartridge. After the sample loading, air was passed through the cartridge for $10 \mathrm{~min}$ in order to remove all of the residual traces of the solution. Then, the cartridge was washed with $250 \mu \mathrm{L}$ of $50 \mathrm{mmol} \mathrm{L}^{-1}$ MES buffer, $\mathrm{pH} 4.5$, ultrapure water, methanol, acetonitrile, acetone, tetrahydrofuran or ethyl acetate. Air was passed through the cartridge for $10 \mathrm{~min}$ to remove all of the residual traces of the washing solution and the retained ciprofloxacin was eluted with $250 \mu \mathrm{L}$ of methanol/acetic acid $9+1 v / v$.

To investigate the effects of the different loading volumes, ciprofloxacin $\left(200 \mathrm{ng} \mathrm{mL}^{-1}\right)$ in synthetic urine was diluted $1+9 v / v$ with $50 \mathrm{mmol} \mathrm{L}^{-1} \mathrm{MES}$ buffer, $\mathrm{pH} 4.5$ and $0.10,0.25$, $0.50,1.00,2.00$ or $5.00 \mathrm{~mL}$ of diluted solution was loaded into the cartridge. Then, air was passed through the cartridge for $10 \mathrm{~min}$ in order to remove all of the residual traces of the loading solution and the retained ciprofloxacin was eluted with $250 \mu \mathrm{L}$ of methanol/acetic acid $9+1 v / v$.

\subsection{MISPE Selectivity}

In order to investigate the selectivity of the optimized extraction protocol in the loading solution, ciprofloxacin was substituted with 7 other fluoroquinolones (danofloxacin, enrofloxacin, levofloxacin, lomefloxacin, moxifloxacin, norfloxacin and sarafloxacin), and chlortetracycline, which was chosen as an unrelated substance. For each of these ligands, a standard solution of $200 \mathrm{ng} \mathrm{mL}^{-1}$ in synthetic urine was diluted $1+9 v / v$ with $50 \mathrm{mmol} \mathrm{L}^{-1}$ MES buffer, $\mathrm{pH} 4.5$ and then $250 \mu \mathrm{L}$ of this diluted solution was loaded into the cartridge. Then, air was passed through the cartridge for $10 \mathrm{~min}$ in order to remove all of the residual 
traces of the solution. The retained ligands were eluted with $250 \mu \mathrm{L}$ of methanol/acetic acid $9+1 v / v$.

\subsection{MISPE of Real Samples}

Blank urine samples collected from four individuals were mixed and then filtered through a $0.22 \mu \mathrm{m}$ polypropylene membrane. The resulting $10 \mathrm{~mL}$ samples were spiked with known amounts of ciprofloxacin (ranging from 2 to $20 \mu \mathrm{g}$ ), or a mixture of ciprofloxacin, danofloxacin and norfloxacin (20 $\mu \mathrm{g}$ each), and then immediately extracted with the optimized protocol reported in Section 2.7. To evaluate the reproducibility of the MISPE protocol, each extraction was repeated five times and the rate of analyte recovery was evaluated as the average of the single values that were measured.

\section{Results}

\subsection{Optimization of the Polymerization Mixture}

The polymerization mixture that has been previously used [26] to prepare nanoMIPs with molecular ciprofloxacin recognition properties was composed of a cross-linking agent ( $\mathrm{N}, \mathrm{N}^{\prime}$-methylene-bis-acrylamide) and two functional monomers, which have the task of interacting with the template through either hydrogen bonds/ion pairs (acrylic acid) or hydrophobic interactions (N-tert-butylacrylamide). These monomers constitute $70 \%$ molar of the mixture, the remaining $30 \%$ of which consists of $\mathrm{N}$-isopropylacrylamide, a thermoresponsive monomer that is useful for the selective detachment of nanoMIPs from the solid phase at the end of the synthesis process. However, since we were not sure that this mixture was optimal for obtaining nanoMIPs that are characterized by a high affinity constant towards ciprofloxacin, we chose to utilize a two-factor central composite $(\mathrm{d}=2, \mathrm{n}=9)$ experimental design by varying the relative quantity of the cross-linker (1-5 $\mathrm{mol} \%$ ) and the functional monomers (acrylic acid, $10-50 \mathrm{~mol} \%$ ) in the polymerization mixtures, keeping unchanged the amount of thermoresponsive monomer.

The binding affinities for ciprofloxacin calculated from Langmuirian binding models (the results of which are reported in Table S2, Supplementary Materials) were fitted against the molar percentages of AA and tBAM by using a six-parameter polynomial model. The response surface, reported in Figure 1, fits quite well with the experimental design points used in the design, with $r^{2}=0.921$ and fit standard error $=0.607$ (see Table S3, Supplementary Materials). Less complex five- or four-parameter designs that were lacking in interaction or quadratic parameters were discarded because they produced response surfaces with lower correlation coefficients $\left(\mathrm{r}^{2}<0.8\right)$ and higher fit standard errors $(>1-2)$, As the polynomial model that was used provides an interaction term between the independent variables (parameter $a_{5}$ in Table S3), the resulting surface shows an obvious saddle shape, with two distinct regions where the binding affinities are higher and an intermediate region with a saddle-centered maximum where the binding affinities are lowest. It should be noted that the formulation of the polymerization mixture previously reported in literature [26] corresponds to a response surface region characterized by high binding constants. For these reasons, several polymers with very different molar composition were potentially suitable to set up a MISPE protocol with, among which we have chosen the formulation P6, which corresponds to nanoMIPs with high affinity $\left(3.00 \pm 0.75 \times 10^{6} \mathrm{M}^{-1}\right)$ and binding site concentration $\left(18.8 \pm 2.9 \mathrm{nmol} \mathrm{g}^{-1}\right)$. 


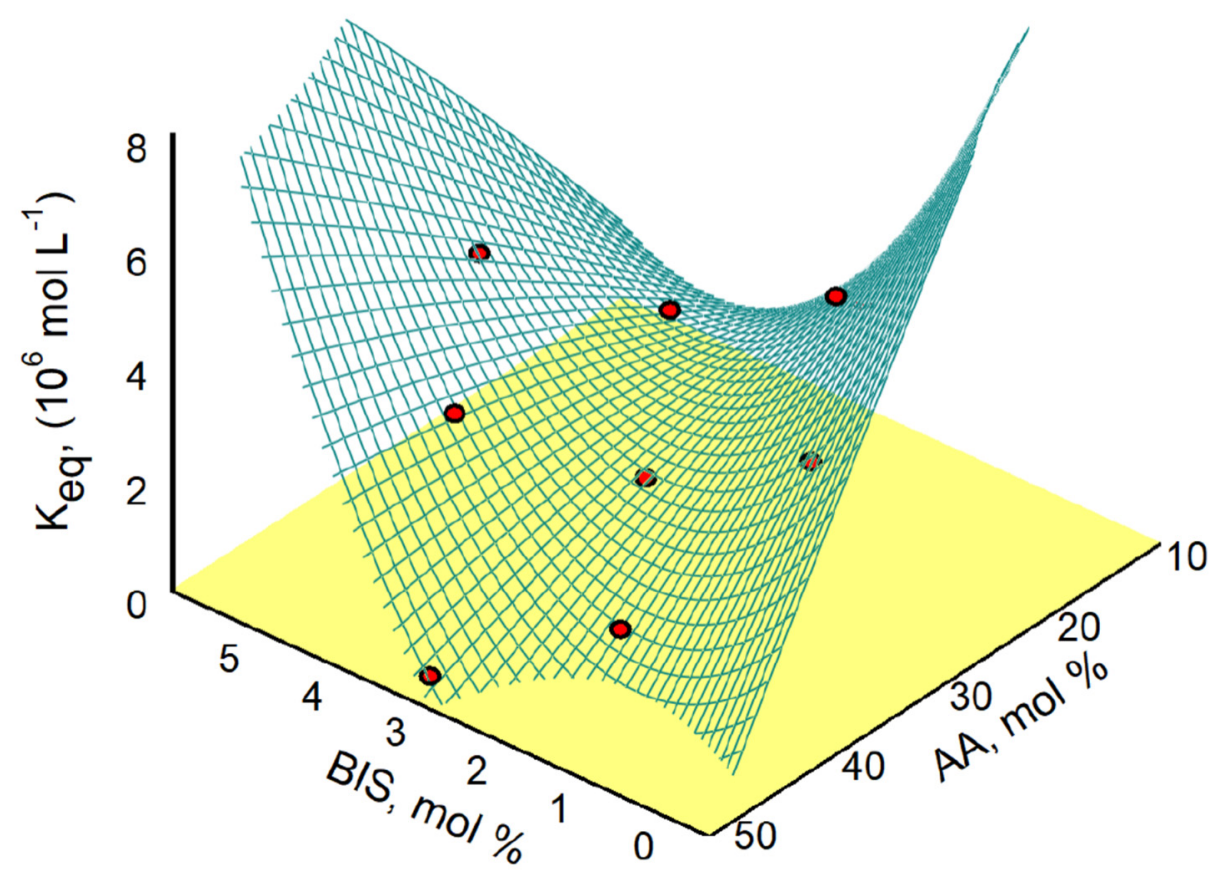

Figure 1. Responding surface of the two-factor central composite design. Red dots: experimental points.

\subsection{Optimization of the MISPE Protocol}

In order to optimize the procedure of fluoroquinolone extraction, we initially focused our attention on the composition of the loading and washing solutions. Synthetic urine containing $200 \mathrm{ng} \mathrm{mL}^{-1}$ of ciprofloxacin was diluted $1+9$ with buffers of different $\mathrm{pH}$ levels. As reported in Figure 2, there is a clear effect of the buffer's $\mathrm{pH}$ on the retention of ciprofloxacin, as the acidic buffer shows a limited loss of analytes while the neutral and basic $\mathrm{pH}$ buffers do not quantitatively retain them. This result confirms the results reported in literature, where ciprofloxacin-binding nanoMIPs show binding constants decreasing when $\mathrm{pH}$ increases [26].

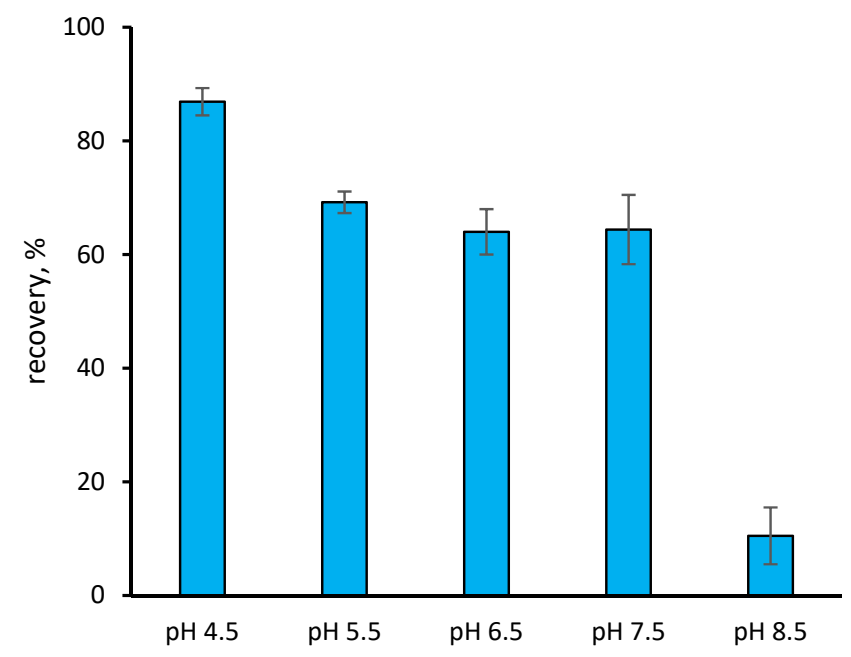

Figure 2. Effect of $\mathrm{pH}$ on loading of $1 \mathrm{~mL}$ of synthetic urine containing $200 \mathrm{ng} \mathrm{mL}^{-1}$ of ciprofloxacin, diluted $9+1 v / v$ with $50 \mathrm{mmol} \mathrm{L}^{-1}$ buffers.

Once optimal loading conditions were defined, we evaluated the effect of the washing solutions; considering $1 \mathrm{~mL}$ of water, an MES buffer at $\mathrm{pH} 4.5$ and organic solvents of decreasing polarity (methanol, acetonitrile, acetone, tetrahydrofuran and ethyl acetate). As reported in Figure 3, none of these solutions, with the exception of the less-polar ethyl acetate, is capable of quantitatively retaining ciprofloxacin. It should be noted that the 
loading buffer is also unable to fully retain the analyte, even if the loss is significantly less $(\sim 25 \%)$ than that of all of the other solutions. As a consequence of this result and taking into account that, by eluting the cartridges loaded with real urine samples with ethyl acetate, the corresponding chromatogram does not show any peak whatsoever, it was therefore decided to omit the washing step, limiting the process to drying the cartridges carefully after loading the sample.

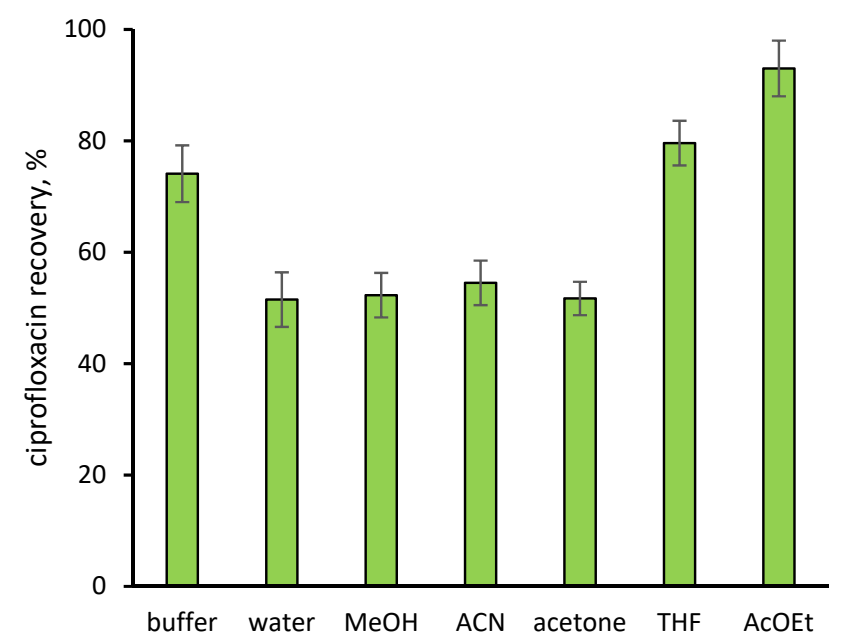

Figure 3. Effect of $1 \mathrm{~mL}$ of washing solutions on retention of ciprofloxacin after loading of $1 \mathrm{~mL}$ of synthetic urine containing $200 \mathrm{ng} \mathrm{mL}^{-1}$ of ciprofloxacin, diluted $9+1 v / v$ with $50 \mathrm{mmol} \mathrm{L}^{-1}$ MES buffer, $\mathrm{pH} 4.5$.

The effect of increasing the loading volumes was evaluated considering samples of synthetic urine spiked with ciprofloxacin at a fixed concentration of $200 \mathrm{ng} \mathrm{mL}^{-1}$ and diluted $1+9 v / v$ with $50 \mathrm{mmol} \mathrm{L}^{-1}$ of MES buffer. The results, reported in Figure 4, show that when the loading volumes are larger than $0.25 \mathrm{~mL}$, the recovery of the analyte drops sharply, becoming very small when $5 \mathrm{~mL}$ of the sample is loaded. This is unexpected, as the nanoMIP P6 that was used to prepare the MISPE cartridges shows a binding site concentration of $18.8 \pm 2.9 \mathrm{nmol} \mathrm{g}^{-1}$. This is a static binding capacity of $\sim 6 \mu \mathrm{g} /$ cartridge of ciprofloxacin, corresponding to 60 times greater than the amount present in $5 \mathrm{~mL}$ of the sample. The cause of this poor loading capacity is unclear, but, in any case, a loading volume of $0.25 \mathrm{~mL}$ ensures a good retention of the analyte in the MISPE protocol reported here.

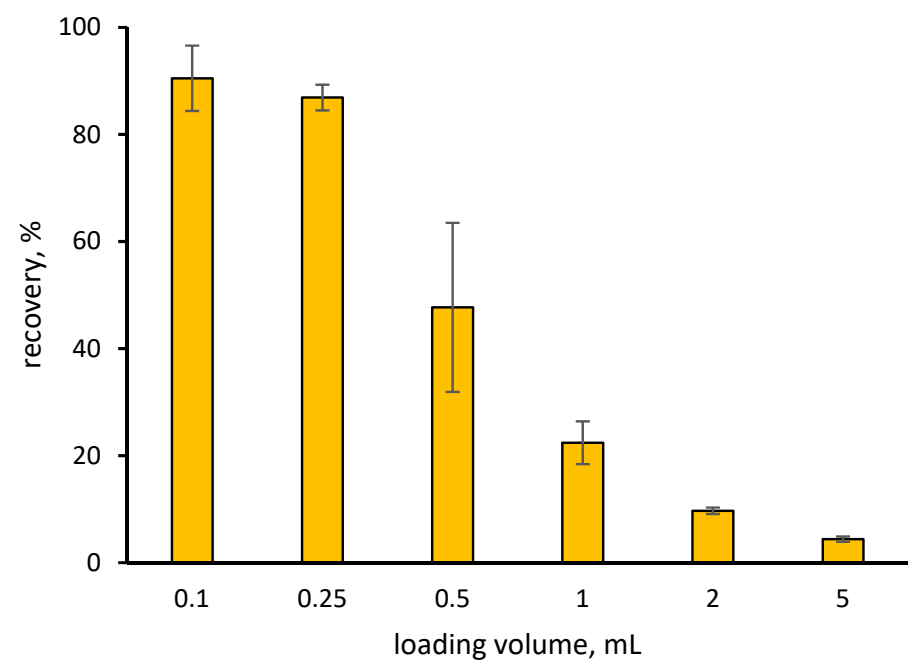

Figure 4. Effect of sample loading volumes on retention of ciprofloxacin, $200 \mathrm{ng} \mathrm{mL}^{-1}$ in synthetic urine, diluted $9+1 v / v$ with $50 \mathrm{mmol} \mathrm{L}^{-1}$ MES buffer, $\mathrm{pH} 4.5$. 


\subsection{MISPE Selectivity}

The selectivity of the optimized MISPE protocol was investigated by extracting several analogues that are related to different generations of antibiotics [29], the molecular structures of which are reported in Scheme 2. They are different from ciprofloxacin by substituents but they share the common fluoroquinolone nucleus.<smiles>O=C(O)c1cn(C2CC2)c2cc(N3CCNCC3)c(F)cc2c1=O</smiles>

ciprofloxacin<smiles>CC1COc2c(N3CCN(C)CC3)c(F)cc3c(=O)c(C(=O)O)cn1c23</smiles><smiles>CCn1cc(C(=O)O)c(=O)c2cc(F)c(N3CCNCC3)cc21</smiles>

norfloxacin<smiles>CN1CC2CC1CN2c1cc2c(cc1F)c(=O)c(C(=O)O)cn2C1CC1</smiles>

danofloxacin<smiles>CCn1cc(C(=O)O)c(=O)c2cc(F)c(N3CCNC(C)C3)cc21</smiles>

lomefloxcin<smiles>O=C(O)c1cn(-c2ccc(F)cc2)c2cc(N3CCNCC3)c(F)cc2c1=O</smiles>

sarafloxacin<smiles>CCN1CCN(c2cc3c(cc2F)c(=O)c(C(=O)O)cn3C2CC2)CC1</smiles><smiles>COc1c(N2CC3CCCNC3C2)c(F)cc2c(=O)c(C(=O)O)cn(C3CC3)c12</smiles>

moxifloxacin<smiles>CN(C)C1C(O)=C(C(N)=O)C(=O)C23OC1CC1c4c(O)ccc(Cl)c4C2(O)C(O)=C(O)C13</smiles>

chlorotetracycline

Scheme 2. Template, fluoroquinolones with similar molecular structure and chlortetracycline. In red: the fluoroquinolone nucleus preserved in all the analytes considered.

The results, reported in Figure 5, show that the imprinted cartridge is able to retain all of the examined fluoroquinolones with recovery rates greater than $80 \%$, while chlortetracycline (an antibiotic substance with a molecular structure completely different from that of fluoroquinolones) is poorly retained. The group selectivity shown by the nanoMIPs can be explained by considering that the positions on the rigid molecular structure of the fluoroquinolone, which presumably are most responsible for the interaction with the binding site, correspond to the positions C3 (carboxyl) and C4 (quinone); these are far from the positions $\mathrm{N} 1$ and $\mathrm{C} 7$ that determine structural differences. It follows that the significant molecular recognition of all fluoroquinolones is essentially determined by the presence of some ubiquitous structures on this class of molecules. These ubiquitous structures are the condensed ring systems that give shape and size to the binding site and the presence of the carboxyl-quinone system in positions $\mathrm{C} 3-\mathrm{C} 4$, which guarantees the same non-covalent interaction mechanism for all molecules. Moreover, it is clear that the basic shape of the ligands is equally important. Chlortetracycline, which possesses a pair of substituents in the C1-C2 positions (similar to the carboxyl-quinone system) but exhibits a radically different condensed ring system, is poorly recognized by nanoMIPs. 


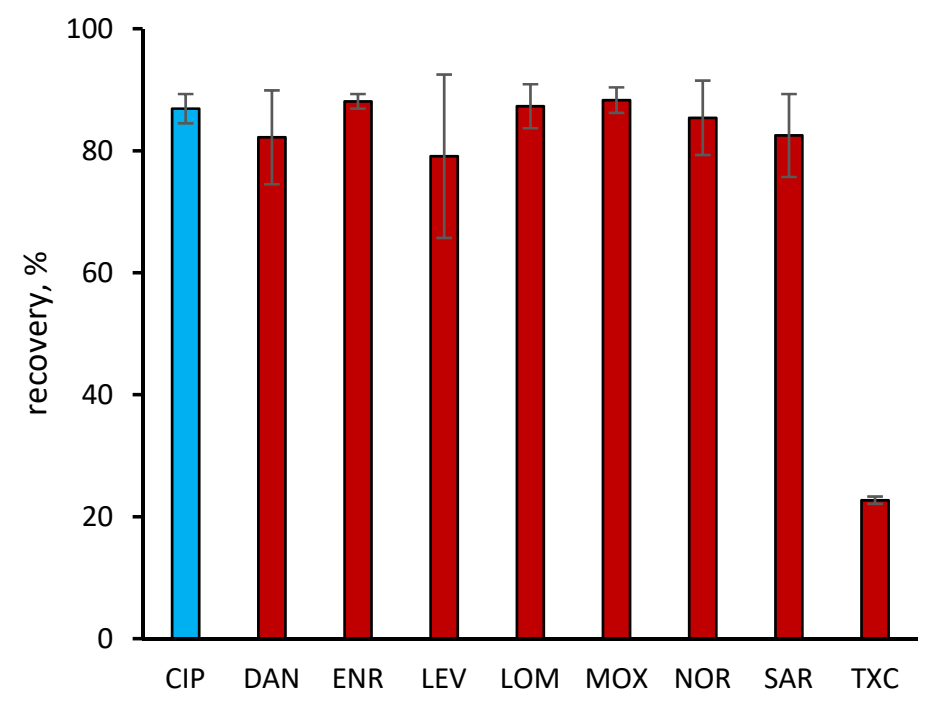

Figure 5. Selectivity after loading of $1 \mathrm{~mL}$ of synthetic urine containing $200 \mathrm{ng} \mathrm{mL}-1$ of analyte, diluted $9+1 v / v$ with $50 \mathrm{mmol} \mathrm{L}^{-1}$ MES buffer, $\mathrm{pH} 4.5$.

\subsection{MISPE of Real Samples}

In order to evaluate the ability of the MISPE cartridges to extract from real samples of human urine, blanks were spiked with known amounts of ciprofloxacin or a mixture of three different fluoroquinolones (ciprofloxacin, danofloxacin, and norfloxacin).

The recovery of ciprofloxacin was determined by comparing the HPLC detector responses of five different urine samples. The recovery rates, reported in Table 1, were determined at five concentration levels of ciprofloxacin (between 0.2 to $2 \mu \mathrm{g} \mathrm{mL}{ }^{-1}$ ), resulting in levels between about $82 \%$ and $85 \%$ with a relative standard deviation $<5 \%$. Moreover, pairwise t-tests performed to compare the recovery rates obtained at different ciprofloxacin concentrations showed no statistical differences between the groups, with $t$-values $(n=5, \alpha=0.05)$ between 0.290 and 0.938 (see Table S4, Supplementary Materials). These results show that the extraction protocol performed well, with good recovery rates and a substantial insensitivity to varying concentration levels.

Table 1. Recovery of ciprofloxacin in human urine after dilution $9+1 v / v$ with $50 \mathrm{mmol} \mathrm{L}^{-1} \mathrm{MES}$ buffer, $\mathrm{pH} 4.5$ and MISPE. Values are the mean $\pm 1 \sigma$ of 5 samples.

\begin{tabular}{cc}
\hline Ciprofloxacin, $\boldsymbol{\mu} \mathbf{g ~ m L}^{-1}$ & Recovery, $\%$ \\
\hline 0.2 & $84.8 \pm 4.4$ \\
0.5 & $82.7 \pm 4.5$ \\
1.0 & $85.4 \pm 4.5$ \\
1.5 & $82.9 \pm 3.6$ \\
2.0 & $85.0 \pm 3.1$
\end{tabular}

$t$-values for pairwise comparison: $0.432(0.2$ vs. $0.5 \mu \mathrm{g} \mathrm{mL}-1), 0.821\left(0.2\right.$ vs. $\left.1.0 \mu \mathrm{g} \mathrm{mL}^{-1}\right), 0.477\left(0.2 \mathrm{vs.} 1.5 \mu \mathrm{g} \mathrm{mL} \mathrm{m}^{-1}\right)$ $0.910\left(0.2\right.$ vs. $\left.2.0 \mu \mathrm{g} \mathrm{mL}^{-1}\right), 0.316\left(0.5\right.$ vs. $\left.1.0 \mu \mathrm{g} \mathrm{mL}^{-1}\right), 0.938\left(0.5\right.$ vs. $\left.1.5 \mu \mathrm{g} \mathrm{mL}^{-1}\right), 0.290\left(0.5\right.$ vs. $\left.2.0 \mu \mathrm{g} \mathrm{mL} \mathrm{gL}^{-1}\right)$, $0.352\left(1.0\right.$ vs. $\left.1.5 \mu \mathrm{g} \mathrm{mL}^{-1}\right), 0.880\left(1.0\right.$ vs. $\left.2.0 \mu \mathrm{g} \mathrm{mL}^{-1}\right), 0.334\left(1.5\right.$ vs. $\left.2.0 \mu \mathrm{g} \mathrm{mL}^{-1}\right)$.

Satisfactory sample clean-up was achieved; this can be seen in the example reported in Figure 6, where the chromatograms of before and after the MISPE of the urine spiked with the fluoroquinolone mixture at a concentration level of $2 \mu \mathrm{g} \mathrm{mL} \mathrm{L}^{-1}$ are reported. When comparing the chromatograms, it can be seen that fluoroquinolones can be detected with some difficulty when a sample of urine is separated directly by reverse phase-HPLC without preliminary MISPE, while the same sample that was analyzed after MISPE shows a cleaner chromatographic trace, where the peaks corresponding to fluoroquinolones can be more easily detected and, as a consequence, quantified. 


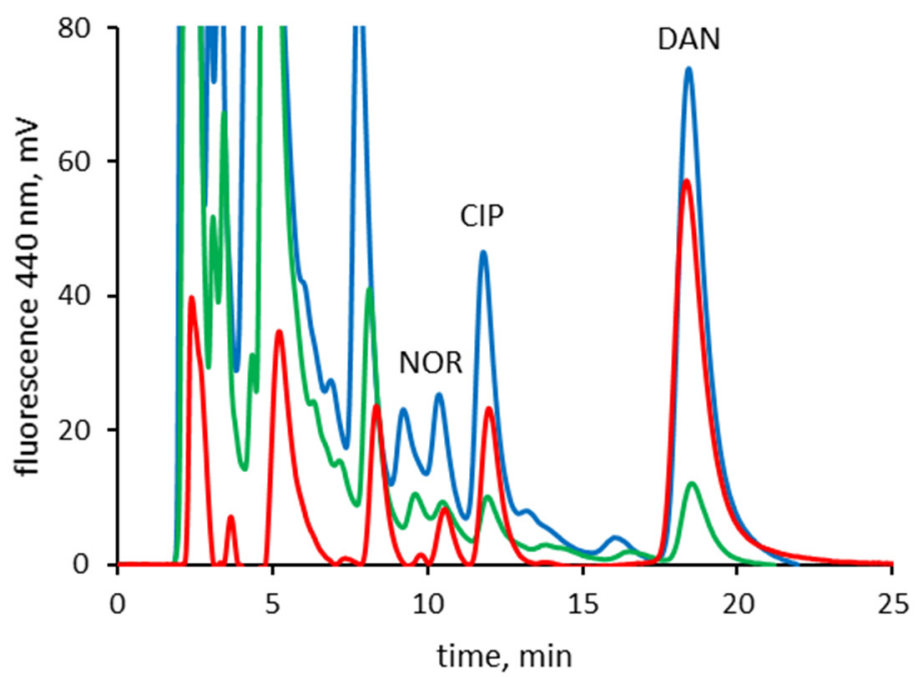

Figure 6. HPLC chromatogram of human urine spiked with ciprofloxacin, danofloxacin, and norfloxacin at final concentration of $2 \mu \mathrm{g} \mathrm{mL}^{-1}$ and diluted $9+1 v / v$ with $50 \mathrm{mmol} \mathrm{L}^{-1}$ MES buffer, $\mathrm{pH}$ 4.5. Blue line: sample injected without MISPE; green line: not retained eluate from MISPE cartridge; red line: eluate from MISPE cartridge.

\section{Discussion}

In this work, we demonstrate that it is possible to use nanoMIPs prepared by solid phase synthesis to develop a MISPE technique that is suitable for real matrices. This result was not taken for granted from the start; in fact, unlike materials prepared through more traditional molecular imprinting techniques, solid phase synthesis allows the preparation of nanopolymers only in very small quantities, at the level of mg per synthesis cycle. Furthermore, the typical dimensions of nanoMIPs obviously do not make their direct use as packing materials for SPE cartridges possible. We, therefore, thought of supporting nanoMIPs on glass microspheres, based on an experimental approach that we have previously used to successfully measure the binding isotherms of this type of nanomaterials $[25,26]$. We also considered it feasible to use very limited amounts of the nanoMIPs by designing their use in this study to align with the use of natural antibodies in the preparation of cartridges for immunoaffinity studies. In fact, even in this case, the typical quantity of antibodies that are covalently bound to the supports is very small. Nonetheless, this small amount does not affect the extraction efficiency of the method [30]. However, it should be noted that the attempt to preconcentrate the samples to further increase the method's sensitivity was not effective, as the ability of MISPE cartridges to retain the analytes with loading volumes greater than $0.25 \mathrm{~mL}$ involves a significant drop in the recovery rate. Since the static binding capacity of the MISPE cartridges used is much greater than the amount of ciprofloxacin that is actually loaded into them, even in the presence of large sample volumes, it is clear that further studies will be necessary to clarify the origin of this behavior and to try to find a possible remedy.

In conclusion, the experimental results reported here show a MISPE/HPLC method allowing the direct extraction of fluoroquinolones from buffered urine samples at the $\mu \mathrm{g} \mathrm{mL} \mathrm{L}^{-1}$ level, with good recovery rates and precision, without interference from the matrix. We believe that these results are, although limited to a small number of analytes in a single real matrix, a good proof-of-concept for the use of nanoMIPs in MISPE methods.

Supplementary Materials: The following are available online at https: / www.mdpi.com/article/ 10.3390/separations8110226/s1, Table S1: molar composition of the prepolymerization mixtures used in the experimental design and resulting binding affinities of the corresponding nanoMIPs. Table S2: nonlinear fitting of Langmuirian binding models for ciprofloxacin-imprinted nanoMIPs. Table S3: nonlinear fitting of 6-parameter polynomial model for the two-factor central composite $(d=2, n=9)$ experimental design. 
Author Contributions: Conceptualization, C.B.; methodology, C.B. and M.C.; validation, C.B. and M.C.; investigation, M.C., S.C. and T.S.; resources, L.A.; data curation, F.D.N.; writing-original draft preparation, M.C.; writing-review and editing, C.B. All authors have read and agreed to the published version of the manuscript.

Funding: This research received no external funding.

Institutional Review Board Statement: Ethical review and approval were waived for this study, due to nature of the biological fluid samples.

Informed Consent Statement: Informed consent was obtained from all subjects involved in the study.

Data Availability Statement: The raw and processed data required to reproduce these findings are available on request.

Conflicts of Interest: The authors declare no conflict of interest.

\section{References}

1. Pichon, V.; Delaunay, N.; Combès, A. Sample Preparation Using Molecularly Imprinted Polymers. Anal. Chem. 2019, 92, 16-33. [CrossRef]

2. Turiel, E.; Martín-Esteban, A. Molecularly imprinted polymers-based microextraction techniques. TrAC Trends Anal. Chem. 2019, 118, 574-586. [CrossRef]

3. Ansari, S. Application of magnetic molecularly imprinted polymer as a versatile and highly selective tool in food and environmental analysis: Recent developments and trends. TrAC Trends Anal. Chem. 2017, 90, 89-106. [CrossRef]

4. Zaidi, S.A. Recent developments in molecularly imprinted polymer nanofibers and their applications. Anal. Methods 2015, 7, 7406-7415. [CrossRef]

5. Zhou, T.; Ding, L.; Che, G.; Jiang, W.; Sang, L. Recent advances and trends of molecularly imprinted polymers for specific recognition in aqueous matrix: Preparation and application in sample pretreatment. TrAC Trends Anal. Chem. 2019, 114, 11-28. [CrossRef]

6. Arabi, M.; Ostovan, A.; Bagheri, A.R.; Guo, X.; Wang, L.; Li, J.; Wang, X.; Li, B.; Chen, L. Strategies of molecular imprinting-based solid-phase extraction prior to chromatographic analysis. TrAC Trends Anal. Chem. 2020, 128, 115923. [CrossRef]

7. Cormack, P.A.; Elorza, A.Z. Molecularly imprinted polymers: Synthesis and characterisation. J. Chromatogr. B 2004, 804, 173-182. [CrossRef]

8. $\quad$ Ellwanger, A.; Karlsson, L.; Owens, P.K.; Berggren, C.; Crecenzi, C.; Ensing, K.; Bayoudh, S.; Cormack, P.; Sherrington, D.; Sellergren, B. Evaluation of methods aimed at complete removal of template from molecularly imprinted polymers. Analyst 2001, 126, 784-792. [CrossRef]

9. Lorenzo, R.A.; Carro, A.M.; Alvarez-Lorenzo, C.; Concheiro, A.; Lorenzo, R.A.; Carro, A.M.; Alvarez-Lorenzo, C.; Concheiro, A. To Remove or Not to Remove? The Challenge of Extracting the Template to Make the Cavities Available in Molecularly Imprinted Polymers (MIPs). Int. J. Mol. Sci. 2011, 12, 4327-4347. [CrossRef]

10. Baggiani, C.; Anfossi, L.; Giovannoli, C. Solid phase extraction of food contaminants using molecular imprinted polymers. Anal. Chim. Acta 2007, 591, 29-39. [CrossRef]

11. Poma, A.; Guerreiro, A.; Whitcombe, M.J.; Piletska, E.; Turner, A.; Piletsky, S.A. Solid-Phase Synthesis of Molecularly Imprinted Polymer Nanoparticles with a Reusable Template-“Plastic Antibodies”. Adv. Funct. Mater. 2013, 23, 2821-2827. [CrossRef] [PubMed]

12. Ambrosini, S.; Beyazit, S.; Haupt, K.; Bui, B.T.S. Solid-phase synthesis of molecularly imprinted nanoparticles for protein recognition. Chem. Commun. 2013, 49, 6746-6748. [CrossRef] [PubMed]

13. Canfarotta, F.; Poma, A.; Guerreiro, A.; Piletsky, S. Solid-phase synthesis of molecularly imprinted nanoparticles. Nat. Protoc. 2016, 11, 443-455. [CrossRef] [PubMed]

14. Altintas, Z.; Guerreiro, A.; Piletsky, S.A.; Tothill, I.E. NanoMIP based optical sensor for pharmaceuticals monitoring. Sens. Actuators B Chem. 2015, 213, 305-313. [CrossRef]

15. Smolinska-Kempisty, K.; Guerreiro, A.; Canfarotta, F.; Cáceres, C.; Whitcombe, M.; Piletsky, S. A comparison of the performance of molecularly imprinted polymer nanoparticles for small molecule targets and antibodies in the ELISA format. Sci. Rep. 2016, 6, 37638. [CrossRef]

16. Chianella, I.; Guerreiro, A.; Moczko, E.; Caygill, J.S.; Piletska, E.V.; Sansalvador, I.M.P.D.V.; Whitcombe, M.J.; Piletsky, S.A. Direct Replacement of Antibodies with Molecularly Imprinted Polymer Nanoparticles in ELISA-Development of a Novel Assay for Vancomycin. Anal. Chem. 2013, 85, 8462-8468. [CrossRef]

17. Tang, S.-P.; Canfarotta, F.; Smolinska-Kempisty, K.; Piletska, E.; Guerreiro, A.; Piletsky, S. A pseudo-ELISA based on molecularly imprinted nanoparticles for detection of gentamicin in real samples. Anal. Methods 2017, 9, 2853-2858. [CrossRef]

18. Altintas, Z.; Abdin, M.J.; Tothill, A.M.; Karim, K.; Tothill, I.E. Ultrasensitive detection of endotoxins using computationally designed nanoMIPs. Anal. Chim. Acta 2016, 935, 239-248. [CrossRef] 
19. López-Puertollano, D.; Cowen, T.; Cruz, A.G.; Piletska, E.; Somovilla, A.A.; Abad-Fuentes, A.; Piletsky, S. Study of Epitope Imprinting for Small Templates: Preparation of NanoMIPs for Ochratoxin A. ChemNanoMat 2019, 5, 651-657. [CrossRef]

20. Altintas, Z. Surface plasmon resonance based sensor for the detection of glycopeptide antibiotics in milk using rationally designed nanoMIPs. Sci. Rep. 2018, 8, 11222. [CrossRef] [PubMed]

21. Moczko, E.; Guerreiro, A.; Cáceres, C.; Piletska, E.; Sellergren, B.; Piletsky, S.A. Epitope approach in molecular imprinting of antibodies. J. Chromatogr. B 2019, 1124, 1-6. [CrossRef] [PubMed]

22. Benito-Peña, E.; Martins, S.; Orellana, G.; Moreno-Bondi, M.C. Water-compatible molecularly imprinted polymer for the selective recognition of fluoroquinolone antibiotics in biological samples. Anal. Bioanal. Chem. 2008, 393, 235-245. [CrossRef] [PubMed]

23. De Oliveira, H.L.; Anacleto, S.D.S.; da Silva, A.T.M.; Pereira, A.; Borges, W.; Figueiredo, E.; Borges, K.B. Molecularly imprinted pipette-tip solid phase extraction for selective determination of fluoroquinolones in human urine using HPLC-DAD. J. Chromatogr. B 2016, 1033-1034, 27-39. [CrossRef]

24. Barahona, F.; Albero, B.; Tadeo, J.L.; Martín-Esteban, A. Molecularly imprinted polymer-hollow fiber microextraction of hydrophilic fluoroquinolone antibiotics in environmental waters and urine samples. J. Chromatogr. A 2018, 1587, 42-49. [CrossRef] [PubMed]

25. Cavalera, S.; Chiarello, M.; Di Nardo, F.; Anfossi, L.; Baggiani, C. Effect of experimental conditions on the binding abilities of ciprofloxacin-imprinted nanoparticles prepared by solid-phase synthesis. React. Funct. Polym. 2021, 163, 104893. [CrossRef]

26. Chiarello, M.; Anfossi, L.; Cavalera, S.; Di Nardo, F.; Artusio, F.; Pisano, R.; Baggiani, C. Effect of Polymerization Time on the Binding Properties of Ciprofloxacin-Imprinted nanoMIPs Prepared by Solid-Phase Synthesis. Polymers 2021, 13, 2656. [CrossRef]

27. Noël, S.; Gasser, V.; Pesset, B.; Hoegy, F.; Rognan, D.; Schalk, I.J.; Mislin, G.L.A. Synthesis and biological properties of conjugates between fluoroquinolones and a N3"-functionalized pyochelin. Org. Biomol. Chem. 2011, 9, 8288-8300. [CrossRef]

28. Di Nardo, F.; Occhipinti, S.; Gontero, P.; Cavalera, S.; Chiarello, M.; Baggiani, C.; Anfossi, L. Detection of urinary prostate specific antigen by a lateral flow biosensor predicting repeat prostate biopsy outcome. Sens. Actuators B Chem. 2020, 325, 128812. [CrossRef]

29. Ball, P. Quinolone generations: Natural history or natural selection? J. Antimicrob. Chemother. 2000, 46, 17-24. [CrossRef]

30. Pichon, V.; Delaunay-Bertoncini, N.; Hennion, M.C. Chapter 33-Immunosorbents in Sample Preparation. In Comprehensive Analytical Chemistry_Sampling and Sample Preparation for Field and Laboratory; Pawliszyn, J., Ed.; Elsevier: Amsterdam, The Netherlands, 2002; Volume XXXVII, pp. 1088-1090. 